J. Kiefer, T. Seeger, T. Dreier, W. Chen, H. Staufer, W. Meier, Laser applications to chemical, security, and environmental analysis: introduction to the feature issue, Applied Optics 58(10) (2019) Lac1-Lac3.

This paper was published in Applied Optics and is made available as an electronic reprint with the permission of OSA. The paper can be found at the following URL on the OSA website:

https://www.osapublishing.org/ao/abstract.cfm?uri=ao-58-10-LAC1.

(C) 2019 Optical Society of America, Inc. 


\title{
Laser Applications to Chemical, Security, and Environmental Analysis: introduction to the feature issue
}

\author{
Johannes Kiefer, ${ }^{1 *}$ Thomas Seeger, ${ }^{2}$ Thomas Dreier, ${ }^{3}$ Weidong Chen, ${ }^{4}$ Hans \\ Stauffer, ${ }^{5}$ Wolfgang Meier ${ }^{6}$ \\ ${ }^{1}$ Engineering Thermodynamics, University of Bremen, 28359 Bremen, Germany \\ ${ }^{2}$ Institute of Engineering Thermodynamics, University of Siegen, 57076 Siegen, Germany \\ ${ }^{3}$ Institute for Combustion and Gas Dynamics - Reactive Fluids, University Duisburg-Essen, \\ 47057 Duisburg, Germany \\ ${ }^{4}$ Laboratoire de PhysicoChimie de l'Atmosphère Université du Littoral Côte d'Opale, 59140 Dunkerque, France \\ ${ }^{5}$ Spectral Energies LLC, Beavercreek, Ohio 45430, USA \\ ${ }^{5}$ Institute of Combustion Diagnostics, German Aerospace Center (DLR), 70569 Stuttgart, Germany \\ *Corresponding author: Thomas.seeger@uni-siegen.de
}

\begin{abstract}
This Applied Optics feature issue on Laser Applications to Chemical, Security, and Environmental Analysis (LACSEA) highlights papers presented at the LACSEA 2018 Sixteenth Topical Meeting sponsored by the Optical Society of America.
\end{abstract}

\section{(c) 2019 Optical Society of America}

Further development of lasers and laser systems is driving applications in optical sensing and analysis in various in various wideranging areas such as the military, industry, security, medicine and chemistry. For analytical purposes in the field, laser sources need to be compact, rugged, tunable in wavelength and of sufficient power to provide quality data for temperature, species composition, and velocity determination. Another aspect in laser-based analytical measurements is the speed with which data can be collected, which is limited - except for constraints in detector electronics - by the repetition rate and wavelength flexibility (if required) of the employed laser systems. Considerable progress has been made in recent years in high repetition-rate pulsed laser systems with pulse energies in the hundreds of milli-joule range that make even optical diagnostics methods with inherently low signal intensity, such as Raman spectroscopy, feasible for long range detection. This special issue is aimed at scientists, engineers, and practitioners interested in understanding the basic principles and diagnostics purposes of a variety of laser-based methods for the quantitative detection and monitoring of essential parameters in probed samples of different origin and aggregate state, i.e., gas, liquid or solid. Its intent is to bring together the development areas of the necessary equipment (lasers, detectors, optics) as well as the methods themselves, and new approaches. But it is also diverse in its applications in chemical, biophysical, combustion, and environmental analysis. As such, interactions within the field often involve a wide diversity and a strong interdisciplinary exchange among researchers. The Sixteenth Topical Meeting on Laser Applications to Chemical, Security, and Environmental Analysis (LACSEA) was the latest in a long line of similar meetings that have included some of the most advanced research in laser spectroscopic analysis and its applications and served as an ideal venue for showcasing exciting new developments in this field.

The Sixteenth biennial LACSEA meeting was held in Orlando/Florida, USA, 25-28 June 2018, as part of the OSA Imaging and Applied Optics Congress and featured a broad range of distinguished papers that focused on recent advances in analytical laser and optical spectroscopy. Approximately 85 outstanding papers were presented at the meeting, including topics such as absorption based gas phase diagnostics, laser-induced breakdown spectroscopy for remote detection of security related species and explosives, new laser and terahertz sources for remote sensing, and micro-optical laser-based systems. Additionally, the technical program included new fundamental research on laser spectroscopic detection techniques for medical, biochemical, and combustion applications. As a result, the number and breadth of the 17 LACSEA papers contained in this special feature of Applied Optics represent an exceptionally wide range of interesting and new research in this expanding field.

With 5 contributions in this field, high-repetition rate diagnostics of combustion systems is a hot topic in the special issue. Fu et al. applied simultaneous high-speed laser-induced incandescence (LII) and stereo particle imaging velocimetry (Stereo-PIV) to an ethyleneair diffusion flame, which was acoustically stimulated with a frequency of $100 \mathrm{~Hz}$ at various amplitudes. The soot distribution and velocity fields were measured at $20 \mathrm{kHz}$ laser repetition rate. The LII signal was calibrated by the pulse-to-pulse laser energy variation and it has been observed that the soot regions extend along the central axis of the flame and shrink radially under acoustic forcing compared with the unforced flame. Gao et al. combined PIV and planar laser-induced fluorescence (PLIF) to study the response of a laminar diffusion dimethyl-ether flame forced by an acoustic field. A repetition rate of $10 \mathrm{kHz}$ from a burst mode laser was sufficient to visualize flame/vortex interactions. For this purpose, PLIF of formaldehyde, which is a marker of the early-stage fuel decomposition, was carried out. The obtained phase-resolved and time-averaged velocity distributions and vortex images indicate that the amplitude of the acoustic excitation has pronounced effects on the flame via modifying the local heat release. Wang et al. studied an unconfined lean premixed swirling flame under external acoustic excitation in order gain information about thermo-acoustic instabilities. PIV and formaldehyde PLIF were applied at $20 \mathrm{kHz}$ repetition rate to visualize the time-resolved flow-field and the transient flame response to the acoustic 
perturbation. The effects of Reynolds number, Strouhal number, and the acoustic modulation amplitude on the swirling flame were investigated. The results indicate that the Strouhal number has a notable effect on the periodic movements of the inner recirculation zone of the flame. Arndt et al. performed high-speed OH PLIF imaging to study auto-ignition processes in the DLR Jet-in-Hot-Coflow Burner (DLR JHC). The facility was used to inject a turbulent methane jet into the hot exhaust gas of a lean hydrogen/air flame and to establish a steady state jet flame. The fuel could be injected in a transient manner, which led to the observation of an auto-igniting jet. It was possible to evaluate the formation of auto-ignition kernels during the transient fuel injection utilizing time-series of the $\mathrm{OH}$ concentration distributions. It is shown that the $\mathrm{OH}$ concentration levels and their statistics can be used to characterize the chemical state of the reacting flow and to distinguish between auto-ignition and flame propagation. Jiang et al. demonstrated simultaneous measurements of mixture fraction and flow velocity using planar Rayleigh scattering at $100 \mathrm{kHz}$ repetition rate. The image sequences of the mixture fraction distribution were directly derived from the data. Moreover, a $2 \mathrm{D}$ instantaneous flow velocity field was obtained from an optical-flow-based analysis of the Rayleigh scattering images. The mixture fraction and flow velocity field were further analyzed and the result for the centerline showed a good agreement of the mixture fraction with the scaling law.

Further applications of optical diagnostics to reacting and non-reacting gas-phase systems involved a variety of techniques. Weller et al. compared two different Raman spectroscopy approaches for gas analysis. The conventional free space and Cavity-Enhanced Raman Spectroscopy (CERS) were used to measure nitrogen and oxygen in ambient air. The comparison included the real time analysis capabilities, the possibilities to use low power diodes and to make the technique affordable and compact. A comprehensive model for estimating the photon emission for both setups is presented and the trade-offs in how to organize the cavity geometry for maximum gain relative to free space is discussed. The obtained results can serve as a quick guide on how to use low power continuous wave lasers in a cavity setup to obtain enhanced Raman scattering. Bürkle et al. present a comparison of two tunable diode laser absorption spectroscopy (TDLAS) strategies for measuring the residence time distribution in combustion chambers. For this purpose, $\mathrm{HCl}$ was injected and subsequently detected by TDLAS. Moreover, the two methods were applied to gas and solid-fuel combustion processes. Eventually, the experimentally more challenging pulse-injection was found to outperform the commonly used step-injection. Williamson et al. present a laser-induced breakdown spectroscopy (LIBS) method for measuring the temperature in a single shot. They utilize the fact that the breakdown threshold of a low-pressure gas strongly depends on density effects. Hence, monitoring the time-resolved breakdown event with respect to the laser pulse allows deriving temperature. The new method is compared to other thermometry techniques utilizing laser-induced breakdown. Proof-of-concept experiments were carried out along the height of a lean methane-air and a rich propane-air McKenna flame. Reference measurements with radiation and convection corrected thermocouple readings were in good agreement. McCord et al. utilized the chemiluminescence emissions from methane/air flames at elevated pressure to determine the fuel/air ratio. The experiments were carried out in a Hencken burner at pressures of 1-5 bar. The emission spectra were used to investigate the effects of pressure on the $\mathrm{OH}^{*}(308 \mathrm{~nm}), \mathrm{CH}^{*}(430 \mathrm{~nm})$, and $\mathrm{C}_{2}{ }^{*}(500 \mathrm{~nm})$ emissions. It was found that both the $\mathrm{OH}^{*} / \mathrm{CH}^{*}$ and the $\mathrm{C}_{2}{ }^{*} / \mathrm{CH}^{*}$ ratios are proportional to the fuel/air ratio at atmospheric pressure. However, while $\mathrm{C}_{2}{ }^{*} / \mathrm{CH}^{*}$ remains roughly linear to the fuel/air ratio, the $\mathrm{OH}^{*} / \mathrm{CH}^{*}$ ratio becomes highly nonlinear at elevated pressure.

Several papers deal with the physical and physicochemical fundamentals of laser combustion diagnostics. Milde et al. compared the spectral excitation behavior of methane in the mid infrared (MIR) for different excitation sources in view of sensor applications. This was done as the MIR wavelength regime promises better gas detection possibilities than the near-IR or the visible region. Interband cascade (IC) and quantum cascade (QC) based lasers were used as they emit at wavelengths, where the methane exhibits strong absorption transitions. The comparison was done by analyzing the performance of two spectroscopy techniques, TDLAS and Quartz Enhanced PhotoAcoustic Spectroscopy (QEPAS). Wang and Kulatilaka studied high-pressure effects in femtosecond two-photon laser-induced fluorescence (fs-TPLIF) of CO. The fluorescence emission spectrum broadened marginally at the highest pressure of 20 bar. Therefore it was concluded that the broadening can be neglected in most cases. The sub-quadratic dependence of the CO fs-TPLIF signal on the laser fluence increased with the pressure. Moreover, the signals were found to decay slower compared to earlier ns-TPLIF measurements at elevated pressure. Furthermore, pressure effects in different quenching gases were studied using fixed $\mathrm{CO}$ mole fractions and number densities. Overall, the results show that fs-TPLIF is a promising diagnostics tool for CO detection in practical combustion systems at elevated pressure. Other studies were concerned with line broadening effects in coherent anti-Stokes Raman scattering (CARS) spectroscopy. Jiang et al. measured $\mathrm{CO}_{2}$ S-branch linewidths and their broadening in the presence of $\mathrm{O}_{2}, \mathrm{Ar}$, and $\mathrm{C}_{2} \mathrm{H}_{4}$. Hsu et al. studied the Raman line broadening of the S-branch of $\mathrm{CO}$ under perturbation from $\mathrm{CO}, \mathrm{N}_{2}$, and $\mathrm{CO}_{2}$. Meißner et al. studied line broadening effects in nitrogen and nitrogen-oxygen mixtures to unravel the impact of $\mathrm{N}_{2}-\mathrm{N}_{2}$ and $\mathrm{N}_{2}-\mathrm{O}_{2}$ collisions.

Finally yet importantly, two papers deal with diagnostics of media containing condensed phase matter. Yang and Johnson present a dye visualization experiment as an analogy of X-ray angiographic imaging of blood vessels. The velocimetry technique is demonstrated in a pulsatile tubing flow. For the same flow condition, a digital PIV system was also employed to measure the same flow field in the middle plane. The purpose of the study was to determine the accuracy of the velocity field estimation from the transmittance-based twodimensional projection image of the three-dimensional volumetric flow field by dye visualization. Compared to the PIV results in the middle plane, the averaged velocity magnitude from the dye visualization measurement was underestimated by about $16-24 \%$ in the central region and by about $29-43 \%$ in the outer region of the tube. O'Neil et al. investigate the use of ultrashort-pulse LIBS for detecting airborne metals during the reaction of energetic materials. They demonstrate the detection of metallic particles in the hot flame zone of solid propellant strands burning in the atmosphere. The fs-LIBS apparatus could detect all metallic additives, whereas a previous ns- 
LIBS scheme under comparable conditions was able to detect only higher concentrations of aluminum. Moreover, it is shown that there is a linear relationship between the percentage of laser shots detecting a LIBS signal and the mass percentage of aluminum initially present in the strands.

Over the years the LACSEA meeting has become one of the premier must-attend meetings in the field of laser-based spectroscopic analytics, especially for applications associated with combustion diagnostics, chemical analysis, environmental monitoring, and trace species detection related to security and explosives surveillance. Consequently, the next LACSEA meeting is already being planned for June 2018 in Vancouver, Canada, and we invite you to participate in this stimulating experience and to contribute with your recent results.

Finally, the feature editors thank the authors and reviewers for their exceptional contributions, which allowed this special feature issue to be completed on time. Further, we want to acknowledge the outstanding help of the OSA staff. 